\title{
Noncaseating Pulmonary Granulomas Associated with Small Cell Carcinoma of the Lung
}

JOSEPH P. LYNCH, III, M.D. ANDREW FLINT, M.D.

DONALD M. MILLER, M.D., Ph.D. JOSEPH C. FANTONE, M.D.

Ann Arbor, Michigan
From the Divisions of Pulmonary and Critical Care Medicine and Hematology-Oncology Department of Internal Medicine and Department of Pathology, University of Michigan Medical Center, Ann Arbor, Michigan. Requests for reprints should be ad'dressed to Dr. Joseph P. Lynch, III, Pulmonary and Critical Care Medical Division, Box 55, S11306, 1405 East Ann Street, Ann Arbor, Michigan 48 109. Manuscript accepted May 15, 1984.
Noncaseating pulmonary granulomas are rarely assoclated with primary carcinoma of the lung. The patient described herein presented with constlitutional symptoms and nadular pulmonary inflltrates associated with noncaseating granulomas without evident neoplasm in both transbronchial and open lung biopsy specimens. Despite corticosieroid therapy for presumed sarcoidosis, chest roentgenographic findings worsened and repeated transbronchial biopsy 12 months after the onset of initial symptoms revealed small cell carcinoma of the lung. Twenty-two months after initiation of chemotherapy, the patient is well with no evidence for carcinoma.

Sarcoid-like granulomatous reactions have been observed in association with malignancy, particularly lymphoma, and can be confusing diagnostically. Granulomas in response to solid tumors, when noted, have usually been confined to regional lymph nodes, and rarely involve pulmonary parenchyma. Previous reports have emphasized granulomatous reactions within lymph nodes as incidental findings at necropsy or operation in patients with malignancy but with no clinical evidence for sarcoidosis [1-4]. We describe a patient presenting with an exuberant granulomatous reaction in the lung and severe constitutional symptoms in whom the diagnosis of small cell carcinoma of the lung was subsequently established. Initial corticosteroid therapy for sarcoidosis failed to alter the pulmonary parenchymal process, but chemotherapy for small cell carcinoma led to marked clinical and roentgenographic improvement. Although the association of noncaseating granulomas with small cell carcinoma of the lung is not unique, the extensive granulomatous reaction and severe constitutional symptoms mimicking sarcoidosis are distinctly unusual.

\section{CASE REPORT}

This 66-year-old nonsmoking black man was in excellent health until April 1981 when malaise, anorexia, fatigue, fever, night sweats, headache, dyspnea, and diffuse pulmonary infiltrates developed. Physical examination, blood studies, and an evaluation that included upper gastrointestinal series, barium enema, bone scanning, liver-spleen scanning, and fiberoptic bronchoscopy showed unremarkable findings. No diagnosis was established and no therapy was advised. Over the next several months, he remained symptomatic and lost approximately 40 pounds. He was referred to the University of Michigan Medical Center in October 1981 and was admitted. Physical examination was remarkable only for rhonchi at both lung bases. He had daily fevers up to $102^{\circ}$ to $103^{\circ} \mathrm{F}$ throughout his hospitalization. Chest roentgenography demonstrated bilateral nodular infiltrates without hilar or mediastinal lymphadenopathy (Figure 1). Six sputum cytologic tests did not demonstrate 


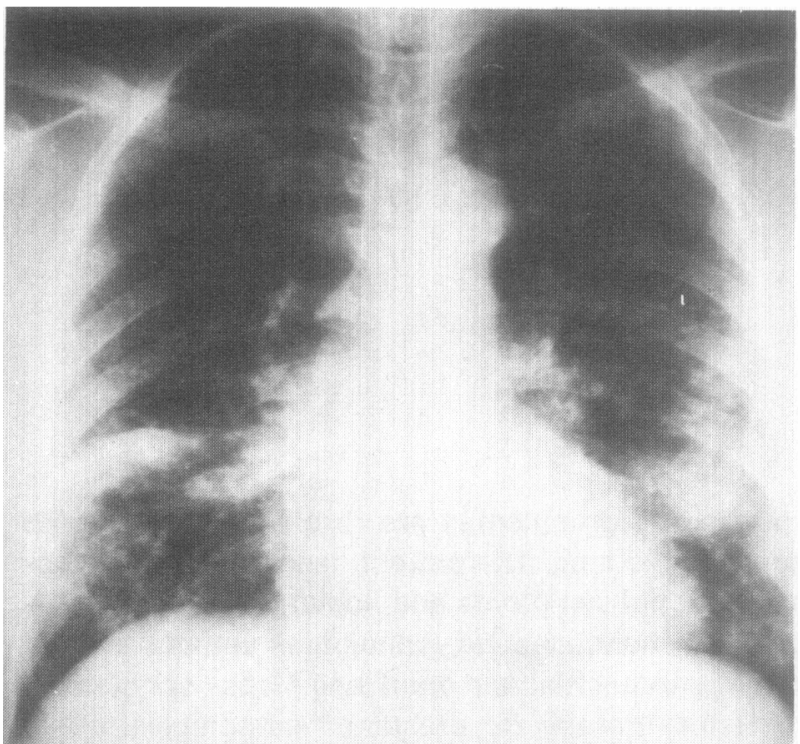

Figure 1. Posteroanterior chest roentgenogram from OCtober 5, 1981 showing diffuse interstitial infiltrates with large nodular densities in the left mid and right lower lung fields. No mediastinal or hilar adenopathy is apparent.

any malignant cells. Flberoptic bronchoscopy and repeated transbronchial biopsy revealed noncaseating granulomas without evidence for malignancy. Bronchial washings and brushings did not show any malignant cells. Results of smears and cultures for acid-fast bacilli and fungi were negative. A percutaneous needle aspirate from a left upper lobe nodular mass showed no neoplasm but revealed multinucleated giant cells consistent with a granulomatous process. A bone marrow aspirate and biopsy specimen were unremarkable with no granulomas or neoplasm. Since the roentgenographic and clinical findings were believed to be atypical for sarcoidosis, thoracotomy with biopsy of the large nodular mass in the left upper lobe was performed. Histopathologic examination revealed extensive noncaseating granulomas with multinucleated giant cells and interstitial fibrosis within the mass, and no evidence for neoplasm (Figure 2). Special stains and cultures of the open lung biopsy specimen showed no acid-fast bacilli or fungi.

In view of the finding of noncaseating granulomas in multiple biopsy specimens with no specific alternative cause, prednisone $60 \mathrm{mg}$ daily was initiated in November 1981 for presumed pulmonary sarcoidosis. After three weeks of prednisone therapy, his symptoms had markedly improved. Over the next several weeks, prednisone was tapered to 20 $\mathrm{mg}$ daily and he remained well, although results of repeated chest roentgenography in January 1982 were unchanged. When the prednisone dose was reduced below $20 \mathrm{mg}$ daily, he noted a gradual return of symptoms.

Because of worsening symptoms, he was readmitted to University Hospital in March 1982. Chest roentgenography showed large nodular densities in the left upper and right lower lung fields, which had worsened since October 1981 and January 1982. Results of physical examination and lab- oratory studies were unremarkable. During this hospitalization, he had daily fever spikes from $102^{\circ}$ to $103^{\circ} \mathrm{F}$. Results of multiple cultures of blood, sputum, urine, and cerebrospinal fluid were negative. He was presumed to have active sarcoidosis and was discharged receiving $60 \mathrm{mg}$ of prednisone daily.

All of his symptoms improved over the next three weeks, but when the prednisone dose was reduced to $40 \mathrm{mg}$ daily. he noted recurrent headaches, malaise, weakness, shortness of breath, fevers, and sweats and was readmitted on April 21, 1982. Physical examination revealed an oral temperature of $101.8^{\circ} \mathrm{F}$ and rhonchi and rales at the right base. Results of the remainder of his examination were normal. Laboratory studies showed a normal hemoglobin level, leukocyte count, erythrocyte sedimentation rate, and angiotensin-converting enzyme level. Serum chemical values included bilirubin 0.4 $\mathrm{mg} / \mathrm{dl}$, serum glutamic oxaloacetic transaminase $27 \mathrm{lU} /$ liter, calcium $10.3 \mathrm{mg} / \mathrm{dl}$, phosphorus $3.4 \mathrm{mg} / \mathrm{dl}$, alkaline phosphatase $119 \mathrm{lU} /$ liter, total protein $5.9 \mathrm{~g} / \mathrm{dl}$, and albumin 3.7 g/dl. Chest roentgenography showed extensive nodular densities in the left upper and right lower lung fields, which had markedly progressed since January 1982 (Figure 3). Results of multiple blood cultures were negative. He continued to have fever spikes up to $103^{\circ}$ and $104^{\circ} \mathrm{F}$. Fiberoptic bronchoscopy showed diffuse erythema of the bronchial mucosa. Bronchial brushings, washings, and transbronchial lung biopsy specimens from the left upper lobe showed dense sheets of malignant cells diagnostic of small cell carcinoma (Figure 4). No granulomas were identified. A bone marrow aspirate and biopsy specimen were within normal limits.

When the diagnosis of small cell carcinoma was established, the previous transbronchial and open lung biopsy specimens from October 1981 were reviewed and multiple sections were recut and examined. Noncaseating granulomas with multinucleated giant cells and fibrosis were again noted, but no neoplastic cells were seen. In order to better delineate the extent of the neoplasm, fiberoptic bronchoscopy was

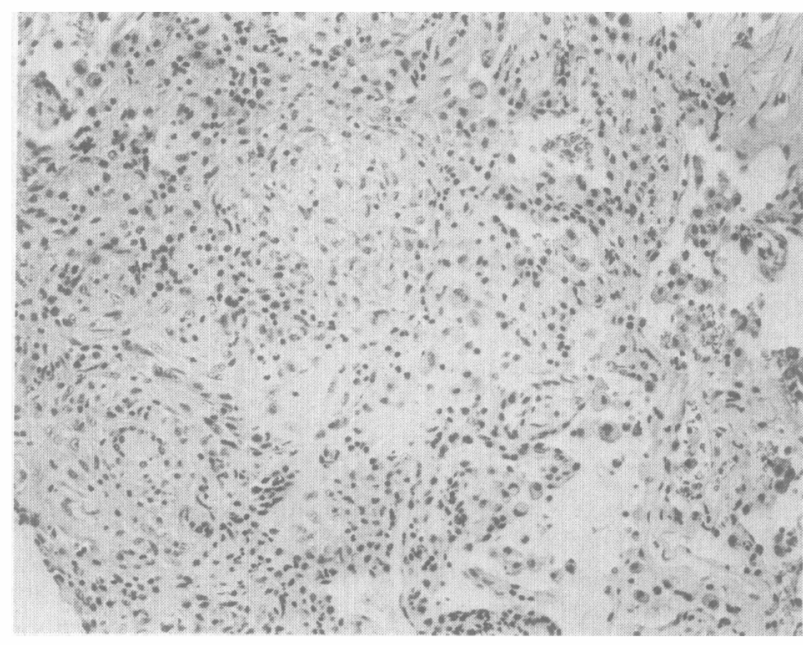

Flgure 2. Section of open lung biopsy specimen shows interstitial non-necrotizing granulomatous inflammation with giant cell formation (hematoxylin and eosin stain; original magnification $\times 125$, reduced by 30 percent). 


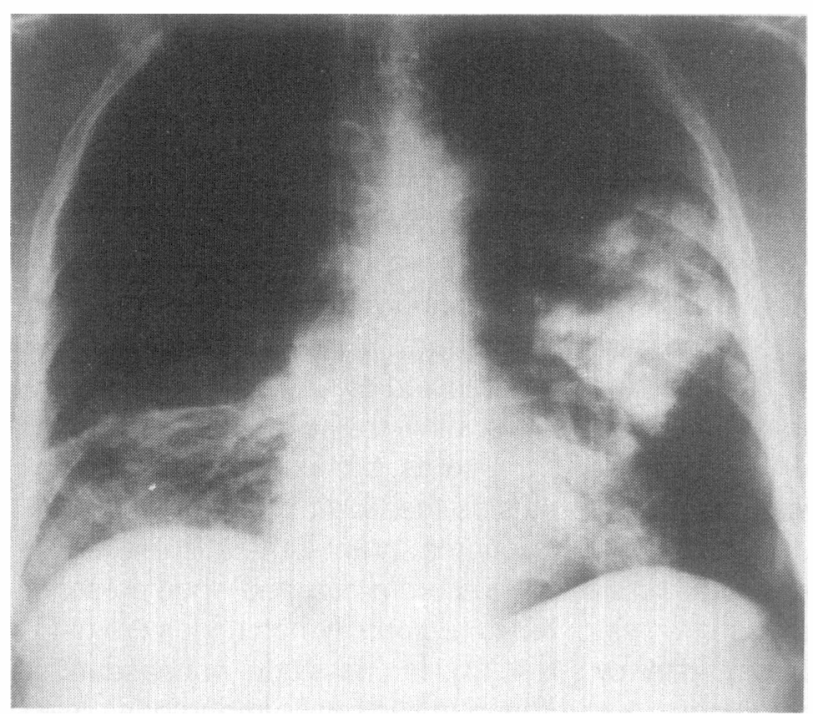

Figure 3. Posteroanterior chest roentgenogram from April 29, 1982 reveals progression of the large nodular masses in the left upper and right lower lung fields since October 1981.

repeated and multiple transbronchial biopsy specimens from the right lower lobe were obtained. Histopathologic examination again revealed small cell carcinoma with no evidence for granulomas. The prednisone dose was maintained at $\mathbf{4 0}$ $\mathrm{mg}$ daily, since lower doses were associated with extensive sweats, fevers, and malaise. Intravenous chemotherapy with cyclophosphamide, vincristine, and doxorubicin was initiated on May 1, 1982. Within five days of initiation of chemotherapy, his fevers and sweats had resolved. Chest roentgenography on May 10, 1982, 10 days after initiation of chemotherapy, showed partial regression in the large nodular densities (Figure 5). His prednisone dose was progressively reduced to $20 \mathrm{mg}$ daily over the next 10 days, and he remained afebrile and without constitutional symptoms.

Over the next nine months, he received 10 outpatient courses of chemotherapy with vincristine doxorubicin, and cyclophosphamide. The dose of prednisone was reduced to $10 \mathrm{mg}$ daily by July 1982 and was discontinued in November 1982. Since initiation of chemotherapy in May 1982, he has gained 42 pounds and has had no recurrence of fever, sweats, chills, or headache. Serial chest roentgenography has shown continued regression of the widespread pulmonic infiltrates (Flgure 6). Chemotherapy was discontinued in February 1983, and he has remained well with no evidence for recurrent neoplasm. Fiberoptic bronchoscopy was repeated in March and August 1983 to determine if residual tumor existed. Results of endoscopic examination were normal, and transbronchial lung biopsy specimens obtained from the left upper lobe were unremarkable with no evidence for neoplasm. He has remained essentially asymptomatic. When the patient was last seen in March 1984, results of physical examination were within normal limits and chest roentgenography demonstrated only mild linear streaking in the left upper and right lower lung fields, consistent with mild fibrosis.

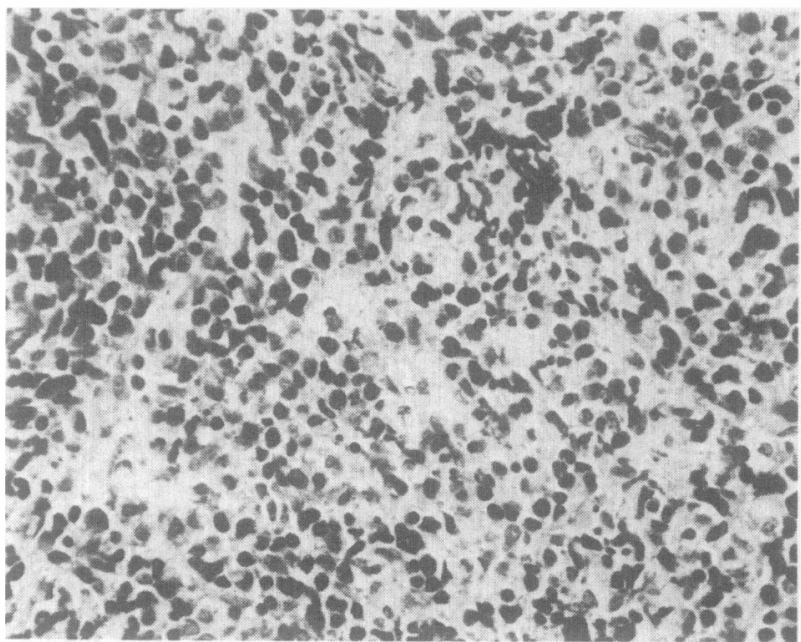

Flgure 4. Section of transbronchial lung biopsy specimen shows diffuse sheets of small atypical cells with inconspicuous nucleoli, coarse chromatin pattern, and high nuclearto-cytoplasmic ratio diagnostic of small cell carcinoma of the lung (hematoxylin and eosin stain; original magnification $X$ 312 , reduced by 30 percent).

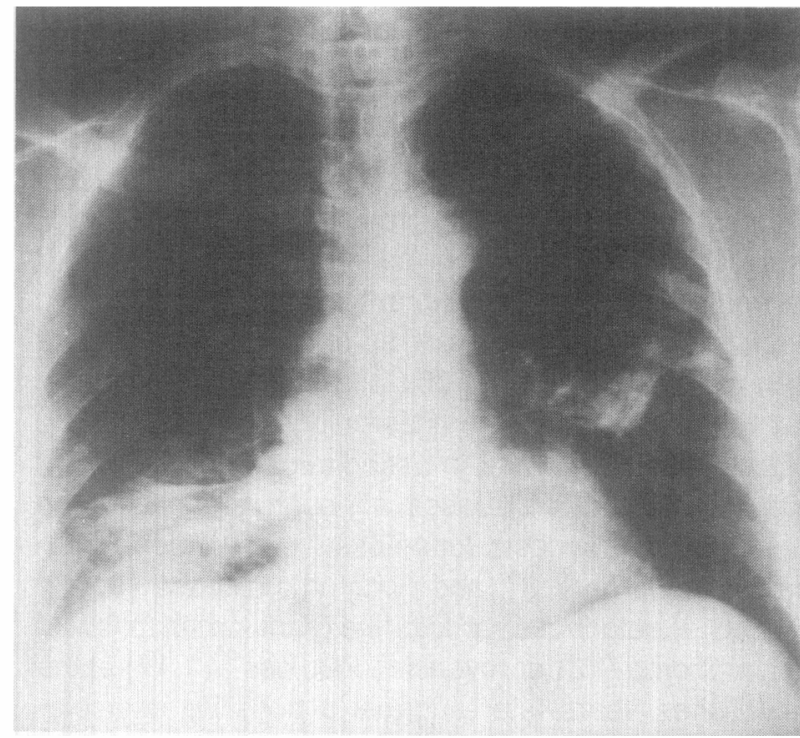

Figure 5. Posteroanterior chest roentgenogram from May 10, 1982, 10 days after initiation of chemotherapy, demonstrating partial regression of left upper lobe mass since April $29,1982$.

\section{COMMENTS}

Sarcoid-like granulomas have been well recognized in association with a variety of malignant neoplasms $[1-4]$, particularly with lymphomas $[5,6]$, and may represent an immunologic response to malignancy. Noncaseating granulomas have been found in liver, spleen, lymph nodes, and bone marrow in 9 to 19 per- 


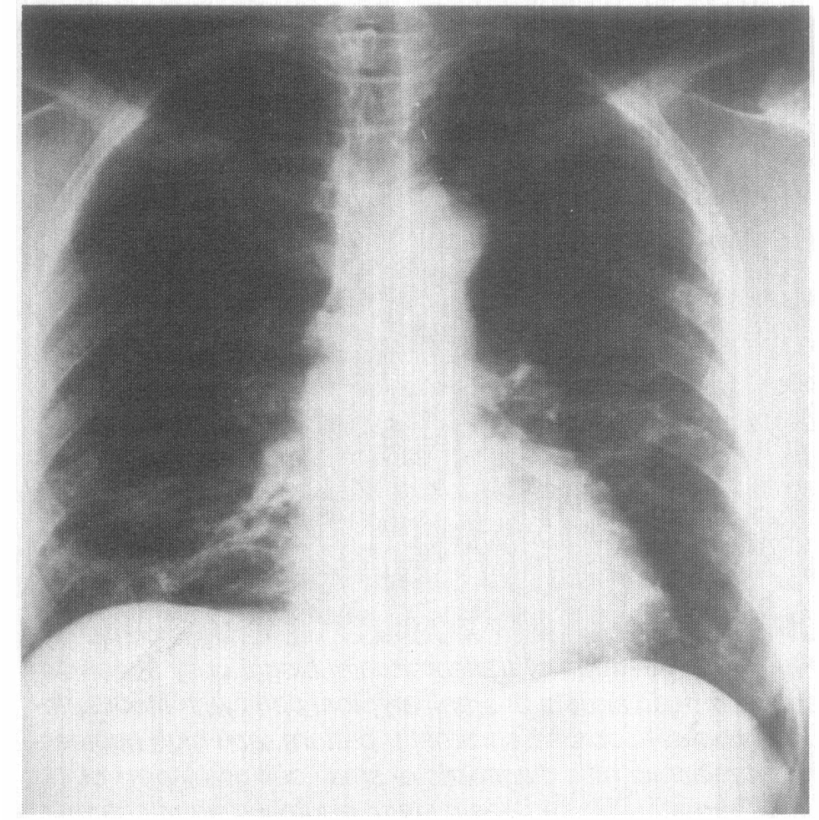

Figure 6. Posteroanterior chest roentgenogram from November 12, 1982 demonstrating nearly complete resolution of left upper and right lower lobe masses since May 1982.

cent of patients undergoing staging laparotomy for Hodgkin's disease [5-9], and have been associated with an improved prognosis [6,8]. Granulomas in association with non-Hodgkin's lymphoma are less common, but noncaseating granulomas in liver, spleen, or lymph nodes were reported in five of 84 patients (6 percent) with non-Hodgkin's lymphoma undergoing staging laparotomy [10], and splenic granulomas were demonstrated in three of 26 (12 percent) patients with non-Hodgkin's lymphoma undergoing splenectomy [7]. Cutaneous granulomas in association with malignant lymphoproliferative diseases, although rare, have been reported with mycosis fungoides $[11,12]$, non-Hodgkin's lymphoma [13], and Hodgkin's disease [9,14]. Reports of cutaneous sarcoid-like granulomas followed by lymphoma [13] or mycosis fungoides $[11,12]$ at the same sites years later suggest a possible interrelationship between these manifestations. No patient described in the aforementioned reports [5-14] had clinical evidence for sarcoidosis or noncaseating granulomas documented within the lung.

Despite the frequent finding of granulomas in malignant lymphoma, histologic confirmation of noncaseating granulomas within lung parenchyma is rare. Brincker [15] described five patients with systemic sarcoidosis among 1,500 patients with lymphoma, but only two had evidence for noncaseating granulomas within lung parenchyma. In both patients, evidence for sarcoidosis existed for eight years or more prior to the development of lymphoma, suggesting that the asso- ciation of these diseases, at least in these instances, was fortuitous. Goldfarb and Cohen [16] described two patients with Hodgkin's disease and noncaseating granulomas at multiple sites, including one in whom open lung biopsy revealed noncaseating granulomas but no evidence for Hodgkin's disease. Buckle [17] described a patient with asymptomatic pulmonary infiltrates on chest roentgenography in whom biopsy of a neck mass six months later revealed non-Hodgkin's lymphoma. Necropsy showed extensive noncaseating granulomas and fibrosis within the lung without evidence for intrapulmonary lymphoma, but extensive lymphoma was documented outside the lungs.

In contrast to lymphoma, granulomatous reactions to solid tumors are far less common, and when present, are usually restricted to regional lymph nodes draining the malignancy $[1-4,18]$. In one study, noncaseating granulomas were found in regional lymph nodes in $\mathbf{8}$ percent of patients with carcinoma of the cervix [19]. In all of the aforementioned studies, noncaseating granulomas were incidental findings at necropsy or operation and were confined to the regional lymph nodes draining the malignancy in all but two patients $[4,18]$.

Granulomatous lymphadenopathy in nodes distant from malignant tumors has also been described. Trump et al [20] described two patients in whom hilar, mediastinal, and paratracheal lymphadenopathy developed following cytotoxic or radiation therapy for testicular carcinoma. In both cases, noncaseating granulomas were demonstrated on lymph node biopsy with no evidence for carcinoma. Sybert and Butler [21] reported hilar and paratracheal adenopathy in a patient who had previously undergone amputation and chemotherapy for osteosarcoma of the femur. Biopsy specimens of a mediastinal node and a pulmonary nodule both revealed noncaseating granulomas without evidence for neoplasm. Geller et al [22] reported noncaseating granulomas on mediastinal node biopsy in a patient with bilateral hilar adenopathy, in whom carcinoma of the testis was found at subsequent orchiectomy. Whether the noncaseating granulomas in these patients represented sarcoidosis or an unusual host response to tumor or antineoplastic therapy is impossible to ascertain.

Granulomatous reactions in association with bronchogenic carcinoma have been described in mediastinal $[2,23,24]$, supraclavicular [25], scalene [24], and hilar $[23,24]$ lymph nodes, and can sometimes obscure the diagnosis of malignancy. Granulomatous reactions within regional nodes were demonstrated in 11 of 325 patients (3.4 percent) with bronchogenic carcinoma described by Jepsen [26] and in 20 of 630 patients (3.2 percent) with bronchogenic carcinoma described by Laurberg [27]. Seventeen of these 20 described by Laurberg had no clinical evidence for sarcoidosis, and 
in all 17 the noncaseating granulomas were confined to regional lymph nodes. Only three patients had noncaseating granulomas within lung parenchyma, one of whom had pulmonary infiltrates consistent with sarcoidosis seven years prior to the diagnosis of malignancy.

Few cases of bronchogenic carcinoma with concurrent noncaseating granulomas involving lung parenchyma have been described. Ellman and Harson [28] reported noncaseating granulomas in lung, spleen, liver, and scalene lymph nodes in a patient with bronchogenic carcinoma of the right lower lobe. Goodbody and Taylor [25] found noncaseating granulomas on supraclavicular lymph node biopsy, but two years later demonstrated squamous cell carcinoma of the lung and noncaseating granulomas within the resected lung tissue and hilar nodes. Schoenfeld et al [29] also reported noncaseating granulomas in resected lung from a patient with squamous cell carcinoma. In another report [23], noncaseating granulomas were demonstrated in subcarinal and right paratracheal nodes in a patient with a left lower lobe mass and bilateral hilar adenopathy. Fourteen months later, thoracotomy revealed fibrosarcoma of the left lower lobe as well as noncaseating granulomas within hilar nodes and lung parenchyma.

Despite a high suspicion for malignancy and an aggressive diagnostic approach, our case presented a diagnostic dilemma, since sarcoid-like granulomas were found in multiple biopsy specimens with no demonstrable tumor. Although it is possible that our patient may have had coincidental pulmonary sarcoidosis and small cell carcinoma, the fact that his carcinoma involved the same areas as the prior granulomatous reactions makes it likely that the noncaseating granulomas represented an immunologic response to tumor. Granulomatous reactions may reflect a host's immune response to mallgnant neoplasm. Activated macrophages in vitro have enhanced tumoricidal activity [30], and tumors with enhanced numbers of macrophages within the stroma have been reported to behave less aggressively [30], supporting the concept of the macrophage as a protective cell. It is possible that the fever, night sweats, and constitutional symptoms seen in our patient, which can also occur with active sarcoidosis, may have represented an intensely activated immune response to neoplasia.

Our patient survived without chemotherapy for 12 months from the onset of symptoms until chemotherapy was initiated in May 1982, which far exceeds the mean survival in patients with untreated small cell carcinoma of the lung [31]. Therefore, it is possible that the exuberant granulomatous reaction in this case exerted a protective role, and was a reflection of response to underlying neoplasm rather than a distinct disease process.

\section{REFERENCES}

1. Anderson R, James DG, Peters PM, Thomson AD: Local sarcoid-tissue reactions. Lancet 1962; I: 1211-1213.

2. Murata Y, Tachibana T, Yugawa E, et al: Pathological study of sarcoid-like lesions associated with malignant neoplasms. In: Iwai K, Hosoda Y, eds. Proceedings of the VI International Conference on Sarcoidosis. Tokyo: University Park Press, 1974; 146-151.

3. Nadel EM, Ackerman LV: Lesions resembling Boeck's sarcoid in lymph nodes draining an area containing a malignant neoplasm. Am J Clin Pathol 1950; 20: 952-957.

4. Symmers W: Localized tuberculoid granulomas associated with carcinoma. Their relationship to sarcoidosis. Am J Pathol 1951; 27: 493-521.

5. Kadin ME, Donaldson SS, Dorfman RF: Isolated granulomas in Hodgkin's disease. N Engl J Med 1970;283: 859-861.

6. Sacks EL, Donaldson SS, Gordon J, et al: Epitheloid granulomas associated with Hodgkin's disease: clinical correlations in 55 previously untreated patients. Cancer 1978; 41: $562-567$.

7. Neiman RS: Incidence and importance of splenic sarcoid-like granulomas. Arch Pathol Lab Med 1977; 101: 518-521.

8. O'Connell MJ, Schimpff SC, Kirschner RH, et al: Epithelioid granulomas in Hodgkin's disease. A favorable prognostic sign? JAMA 1975; 233: 886-888.

9. Whittaker JA, Slater A, Al-ishail SA, et al: An assessment of laparotomy in the management of patients with Hodgkin's disease. Q J Med 1978; 47: 291-301.

10. $\operatorname{Kim~H,~Dorfman~RF:~Morphological~studies~of~} 84$ untreated patients subjected to laparotomy for the staging of nonHodgkin's lymphomas. Cancer 1974; 33: 657-674.

11. Atwood WG, Miller RC, Nelson CT: Sarcoidosis and the malignant lymphoreticular diseases. Arch Dermatol 1966; 94 : 144-151.

12. Schwartz RA, Burgess GH, Boltermann OA, et al: Mycosis fungoides associated with florid sarcoid reactions. J Surg Oncol 1980; 14: 347-357.

13. Kahn LB, Gordon W, Camp R: Florid sarcoid reaction associated with lymphoma of the skin. Cancer 1974; 33: 1117-1122.

14. Randle HW, Banks PM, Winkelman RK: Cutaneous granulomas in malignant lymphoma. Arch Dermatol 1980; 116: 441-443.

15. Brincker H: Sarcoid reactions and sarcoidosis in Hodgkin's disease and other malignant lymphomata. $\mathrm{Br} J$ Cancer 1972; 26: 120-128.

16. Goldfarb BL, Cohen SS: Coexistent disseminated sarcoidosis and Hodgkin's disease. JAMA 1970; 211: 1525-1528.

17. Buckle RM: Reticulosarcoma complicating sarcoidosis. Tubercle 1960; 41: 213-216.

18. Gresham GA, Ackerley AG: Giant cell granulomata in regional lymph nodes of carcinoma. J Clin Pathol 1958:11:244250.

19. Gorton G, Linell F: Malignant tumors and sarcoid reactions in regional lymph nodes. Acta Radiol 1957; 47: 381-392.

20. Trump DL, Ettinger DS, Feldman MJ, et al: 'Sarcoidosis' and sarcoid-like lesions. Their occurrence after cytotoxic and 
radiation therapy of testis cancer. Arch Intern Med 1981; 141: 37-38.

21. Sybert A, Butler TP: Sarcoidosis following adjuvant high-dose methotrexate therapy for osteosarcoma. Arch Intern Med 1978; 138: 488-489.

22. Geller RA, Kuremsky DA, Copeland JS, et al: Sarcoidosis and testicular neoplasm: an unusual association. J Urol 1977; 118: 487-488.

23. Case records of the Massachusetts General Hospital. Case 7-1982. N Engl J Med 1982; 306: 412-420.

24. Gregorie HB, Othersen HB, Moore MP: The significance of sarcold-like, lesions associated with malignant neoplasms. Am J Surg 1962; 104: 577-586.

25. Goodbody RA, Taylor AJ: Sarcoidosis and bronchial carcinoma. Tubercle 1957; 38: 419-421.
26. Jepsen O: Mediastinoscopy. Copenhagen: Scandinavian University Books, 1966.

27. Laurberg P: Sarcoid reactions in pulmonary neoplasms. Scand J Respir Dis 1975; 56: 20-27.

28. Ellman $\mathrm{P}$, Harson $\mathrm{A}$ : The coexistence of bronchial carcinoma and sarcoidosis. Br J Tuberc 1958; 52: 218-222.

29. Schoenfeld $Y$, Avidor $E$, Eldar $M$, et al: Squamous cell carcinoma associated with sarcoidosis in the lung. Oncology 1978; 35: 112-113.

30. Hibbs JB, Chapman HA, Weinberg JB: Regulation of macrophage non-specific tumoricidal activity. In: Van Furth $R$, ed. Mononuclear phagocytes. Functional aspects, part II. The Hague: Martinus Nijhoff, 1980; 1681-1720.

31. Hyde L, Yee L, Wilson R, et at: Cell type and the natural history of lung cancer. JAMA 1965; 193: 140-142. 